\title{
Study of Metal-Organic Nanomaterials Structure by X-ray Crystallography Analysis as the Basis for the Development of Quality Control Methods
}

\author{
Svitlana Bilous ${ }^{1}$, Gregory Dmytriv ${ }^{3}$, Gennady Didikin ${ }^{4}$ Natalia Hudz ${ }^{1}$ Roman Lesyk $^{2}$ and Timothy Kalynyuk ${ }^{1}$ \\ 1. Department of Drug Technology and Biopharmaceutics, Danylo Halytsky Lviv National Medical University, Lviv 79010, Ukraine \\ 2. Department of Pharmaceutical, Organic and Bioorganic Chemistry, Danylo Halytsky Lviv National Medical University, Lviv \\ 79010, Ukraine \\ 3. Department of Inorganic Chemistry, Ivan Franko National University, Lviv 79000, Ukraine \\ 4. Department of Electron-Ray Nanotechnology, E.O.Paton Electric Welding Institute NAS of Ukraine, Kyiv 03150, Ukraine
}

\begin{abstract}
The aim of the work is to study the structure of antimicrobial agents metronidazole and levofloxacin and their nanocompositions with silver nanoparticles obtained by by electron-ray nanotechnology method, and to demonstrate the necessity of x-ray crystalography analysis for developing quality control methods of metal-organic nanomaterials. Structure of antimicrobial agents and their nanocompositions was investigated on automatic powder diffractometer STOE STADI P (MoK $\alpha$-radiation stepping removal method, $2^{\circ} \leq 2 \theta \leq 30^{\circ}$, step scan $-0.02^{\circ}$, scanning at one point $-5-10 \mathrm{c}$ ). For identification of nanocompositions the UV spectrophotometry metod was used.
\end{abstract}

Key words: Quality control methods, x-ray crystalography analysis, metronidazole, levofloxacin, silver nanoparticles.

\section{Introduction}

Now the future of effective antimicrobial preparations is connected with nanostructured materials and nanotechnologies. Results of studies in this direction prove that metal nanoparticles, particularly silver nanoparticles can enhance the action of widely used antibiotics and antifungal preparations and prevent antimicrobial resistance that is promising for pharmaceutical development of new preparations [1-5].

In Danylo Halytsky Lviv National Medical University are conducted studies considering pharmaceutical development of antimicrobial preparations in which as active pharmaceutical ingredients are used nanocompositions of silver with metronidazole and levofloxacine [6].

Studies connected with pharmaceutical

Corresponding author: Svitlana Bilous, associate professor, research fields: drug technology, nanotechnology. development are inextricably linked with the QCMs (quality control methods). Without development of QCM for nanostructured materials, including such indicators as methods of identification and quantitative determination it is impossible to investigate the stability of drugs during storage and accordingly to designate the shelf life of preparation [7]. Therefore, now one of the priorities is to develop QCMs of nanostructured substances that will allow intensifying research of pharmaceutical development based on nanotechnologies.

Complexity of QCMs development for nanostructured materials is connected primarily with the fact that nanomaterials including nanometals created by different developers using different methods have very different structures; they are nanoporous structures nanodispersions (colloids), nanostructured surfaces, nanocrystals, nanoclusters and others [5]. 

as the Basis for the Development of Quality Control Methods

The aim of the work is to study the structure of metal-organic nanomaterials, on example antimicrobial substances metronidazole and levofloxacin and propose the approaches to development of analysis methods.

\section{Materials and Methods}

Silver nanoparticles on the surface of antimicrobial agents was applied by electron-ray nanotechnology method in "Laboratory of Electron-Ray Nanotechnology of Inorganic Materials for Medicine" of E.O.Paton Electric Welding Institute NAS of Ukraine $[8,9]$.

To study the structure of antimicrobial agents and their nanocompositions X-ray crystallography method on automatic powder diffractometer STOE STADI $P$ (MoK $\alpha$-radiation, stepping removal method, $2^{\circ} \leq 2 \theta$ $\leq 30^{\circ}$, step scan $-0,02^{\circ}$, scanning in one point $-5-10$ sec) has been used. To identify nanocompositions of antimicrobial agents with silver nanoparticles has been used UV- spectrophotometry method.

\section{Results and discussion}

The algorithm of metal-organic nanomaterials quality control methods development was worked out; algorithm primarily includes study of the structure of nanomaterials, for example, by X-ray crystallography method [10-12].

Further selection of quality control methods should be based on the results of studies of nanomaterials structures. When the diffraction pattern of initial substances does not change after applying metal nanoparticles can be considered pharmacopoeial methods of analysis for the initial substances as potential methods for quality control of their nanocompositions with silver nanoparticles. If metal nanoparticles incorporated into the structure of antimicrobial substances it is necessary to develop new methods of quality control. For the qualitative and quantitative determination of silver nanoparticles in organometallic nanomaterials it is need to use additional method such as inductively coupled plasma atomic emission spectrometry [13].

\subsection{Comparative Studies of Structure of Antimicrobial} Agents Metronidazole and Levofloxacin and Their Nanocompositions with Silver Nanoparticles

Adding of different amounts of silver nanoparticles to substance metronidazole (metronidazole diffractogram is shown in Fig. 1a) does not change the diffraction pattern (diffractogram of metronidazole with silver nanoparticles in concentrations $0.0034 \%$ and $0.0178 \%$ are shown in Figs. $1 \mathrm{~b}$ and $1 \mathrm{c}$, respectively); this indicates that in structure of the original substance metronidazole no changes.

A similar situation was observed at comparing of levofloxacin diffractogram (Fig. 2a) and levofloxacin with silver nanoparticles in concentrations $0.00119 \%$ and $0.00476 \%$ (diffractograms on Figs. $2 b$ and 2c, respectively).

The results suggest that silver nanoparticles applied on the surface of substances metronidazole and levofloxacin by electron-ray nanotechnology method, do not change the structure of the starting materials, and probably stay on the surface of grains of crystals of the substances. This makes it possible to consider pharmacopoeial methods of analysis for metronidazole and levofloxacin as potential methods for the identification and quantitative analysis of their compositions with silver nanoparticles.

\subsection{Identification of Metronidazole and Levofloxacin} and Their Nanocompositions with Silver Nanoparticles by UV Spectrometry

One of the pharmacopoeia methods for identification and quantification of metronidazole is absorption spectrophotometry in the ultraviolet region.

Solutions of metronidazole and metronidazole with silver nanoparticles were made in accordance with the requirements of pharmacopoeia testing and spectra of absorption were withdrawn in the range from $230 \mathrm{~nm}$ to $350 \mathrm{~nm}$. 

as the Basis for the Development of Quality Control Methods

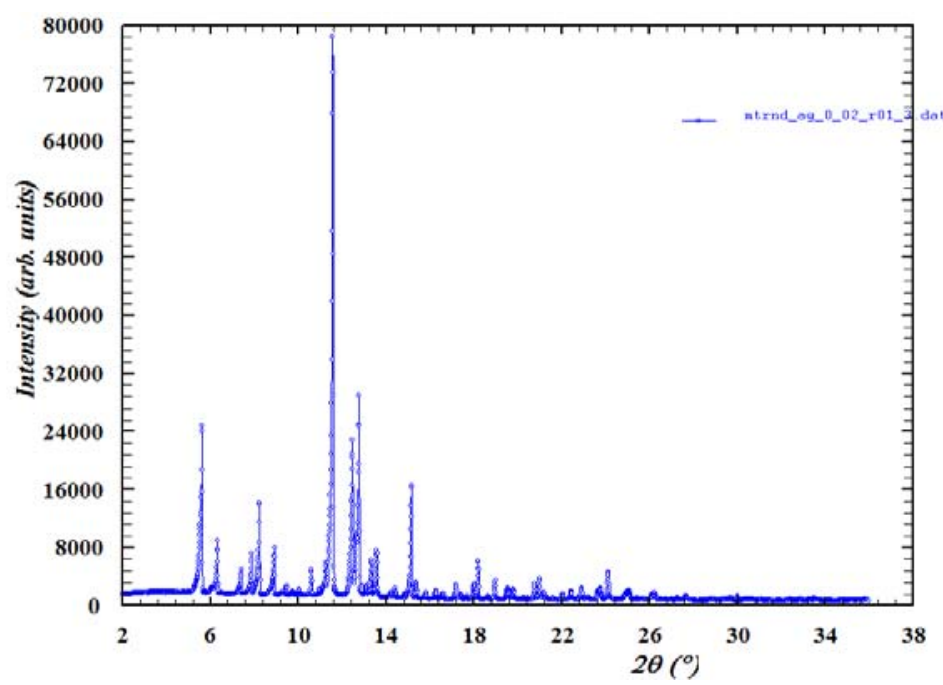

(a)

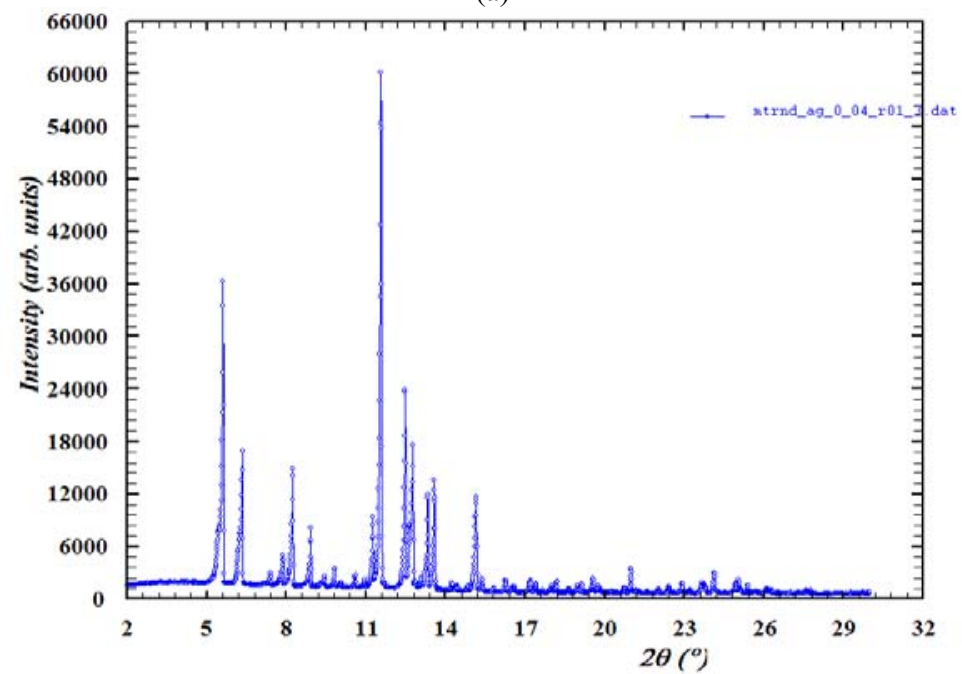

(b)

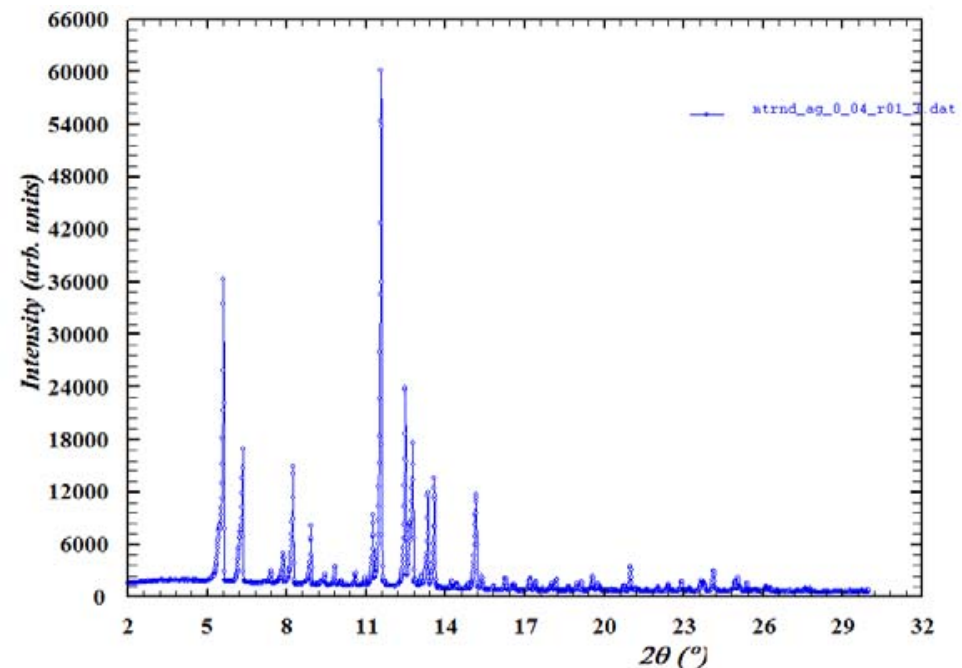

(c)

Fig. 1 a. Diffractogram of metronidazole, b. Diffractogram of metronidazole with silver nanoparticles $(0.0034 \%)$, c. Diffractogram of metronidazole with silver nanoparticles $(\mathbf{0 . 0 1 7 8} \%)$. 
Study of Metal-Organic Nanomaterials Structure by X-ray Crystallography Analysis as the Basis for the Development of Quality Control Methods

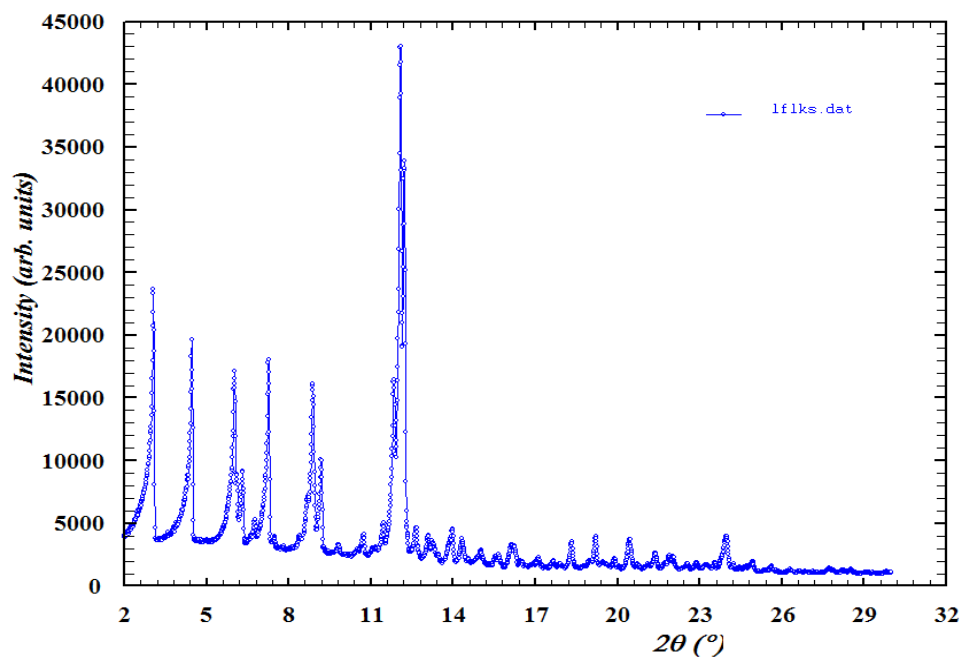

(a)

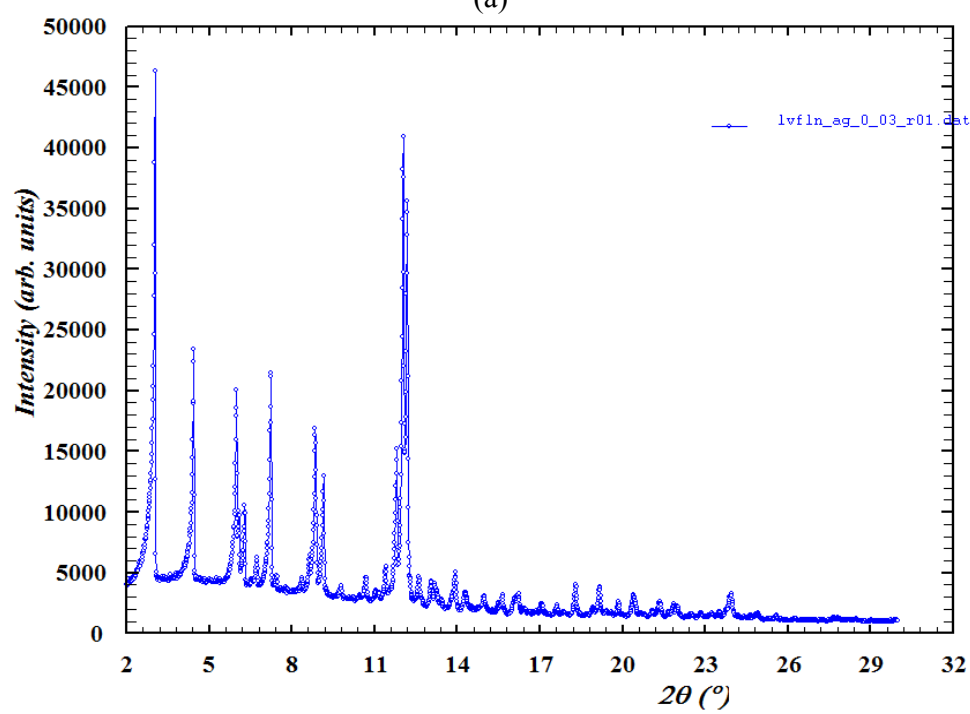

(b)

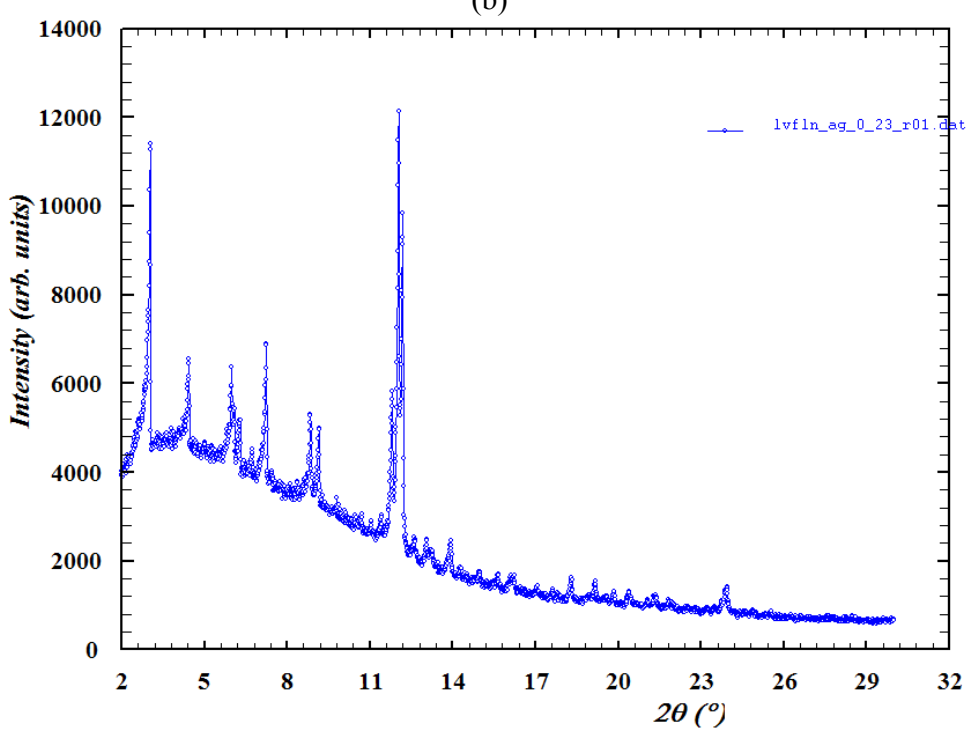

(c)

Fig. 2 a. Diffractogram of levofloxacin, b. Diffractogram of levofloxacin with silver nanoparticles $(0.00119 \%)$, c. Diffractogram of levofloxacin with silver nanoparticles $(\mathbf{0 . 0 0 4 7 6 \% )}$. 
Fig. 3 shows the ultraviolet absorption spectra of metronidazole and metronidazole with silver nanoparticles.

UV absorption spectra of the testing solutions have a maximum at a wavelength $277 \mathrm{~nm}$ and a minimum at a wavelength $240 \mathrm{~nm}$.

Pharmacopoeial method for identification and quantification of levofloxacin also is absorption spectrophotometry in the ultraviolet region.

Figs. $4 \mathrm{a}, 8 \mathrm{~b}$ and $8 \mathrm{c}$, respectively show ultraviolet absorption spectra of levofloxacin and levofloxacin with different concentrations of silver nanoparticles taken in the range from 220 to $310 \mathrm{~nm}$.

UV absorption spectra of the testing solutions have two maxima at a wavelength $293 \mathrm{~nm}$ and $226 \mathrm{~nm}$ and a minimum at a wavelength $263 \mathrm{~nm}$.

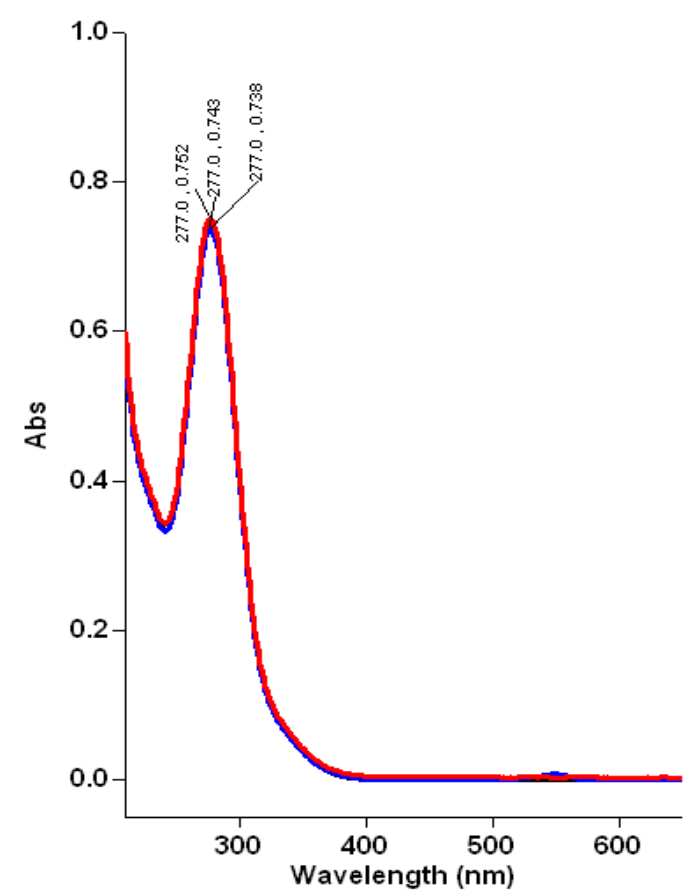

Fig. 3 UV absorption spectra of metronidazole and metronidazole with different concentrations of silver nanoparticles $(0.00119 \%$ and $.00476 \%)$.

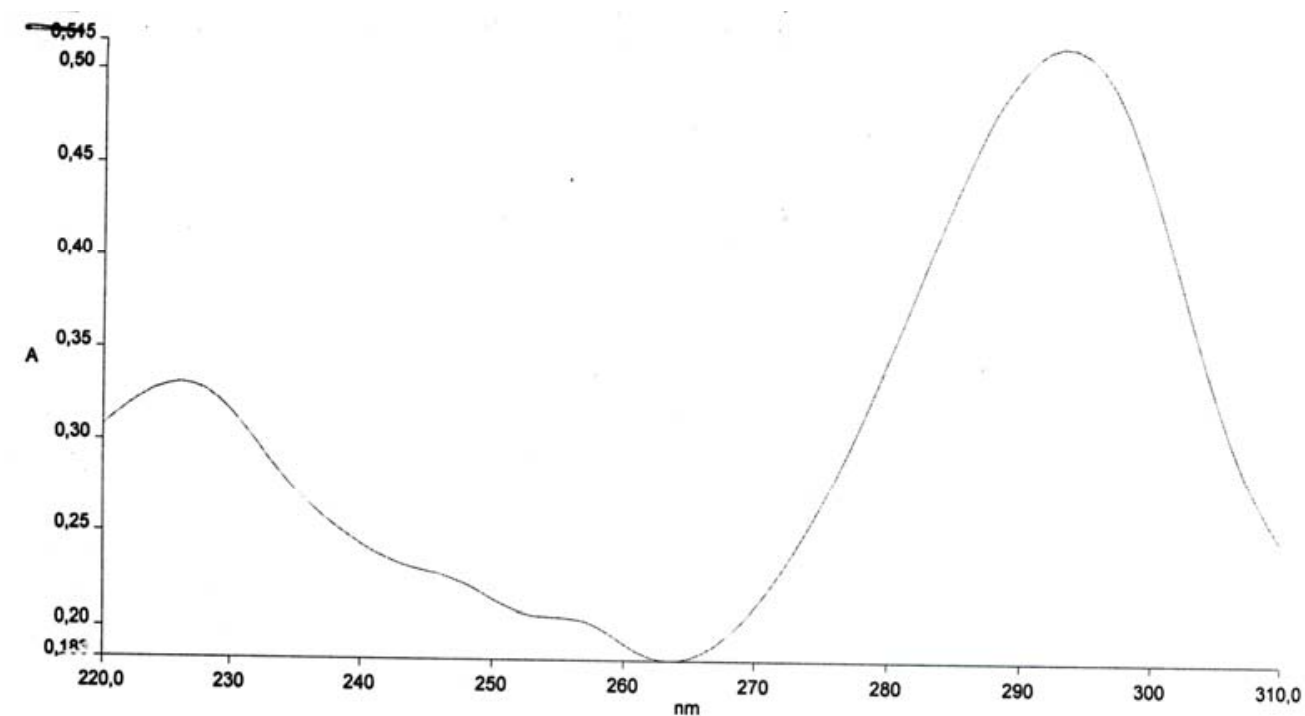

(a) 


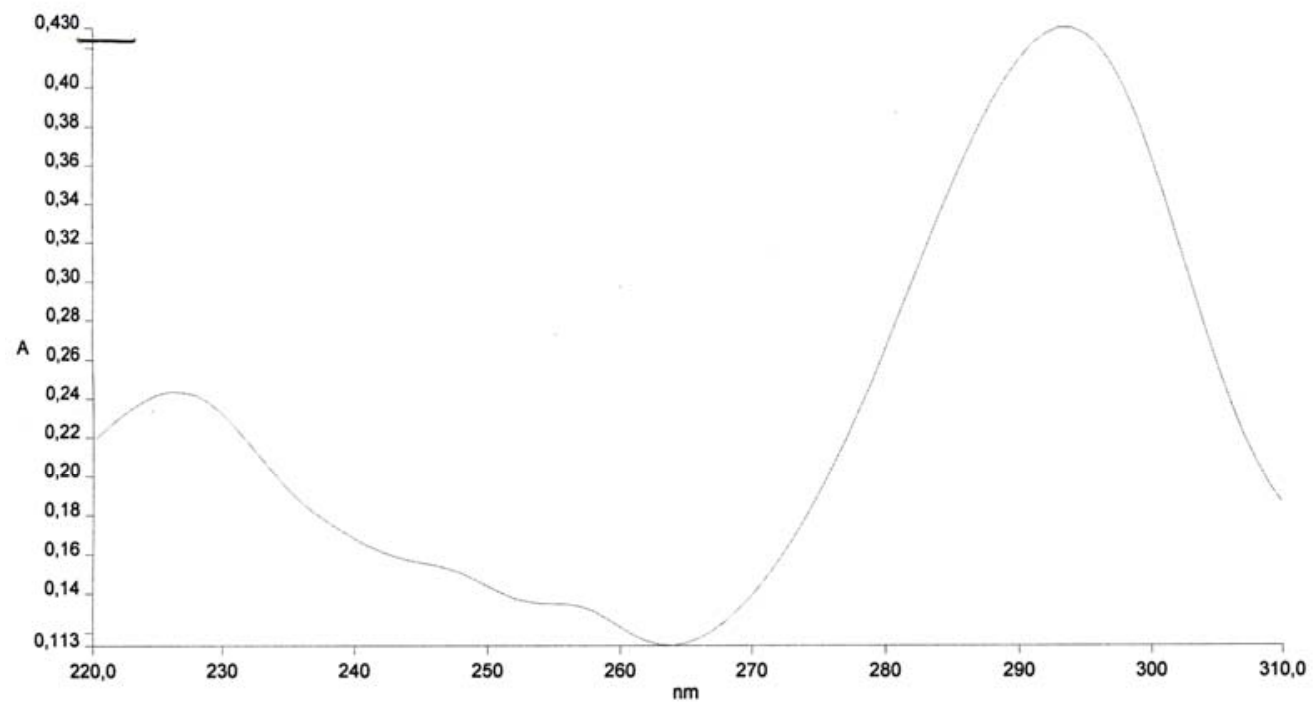

(b)

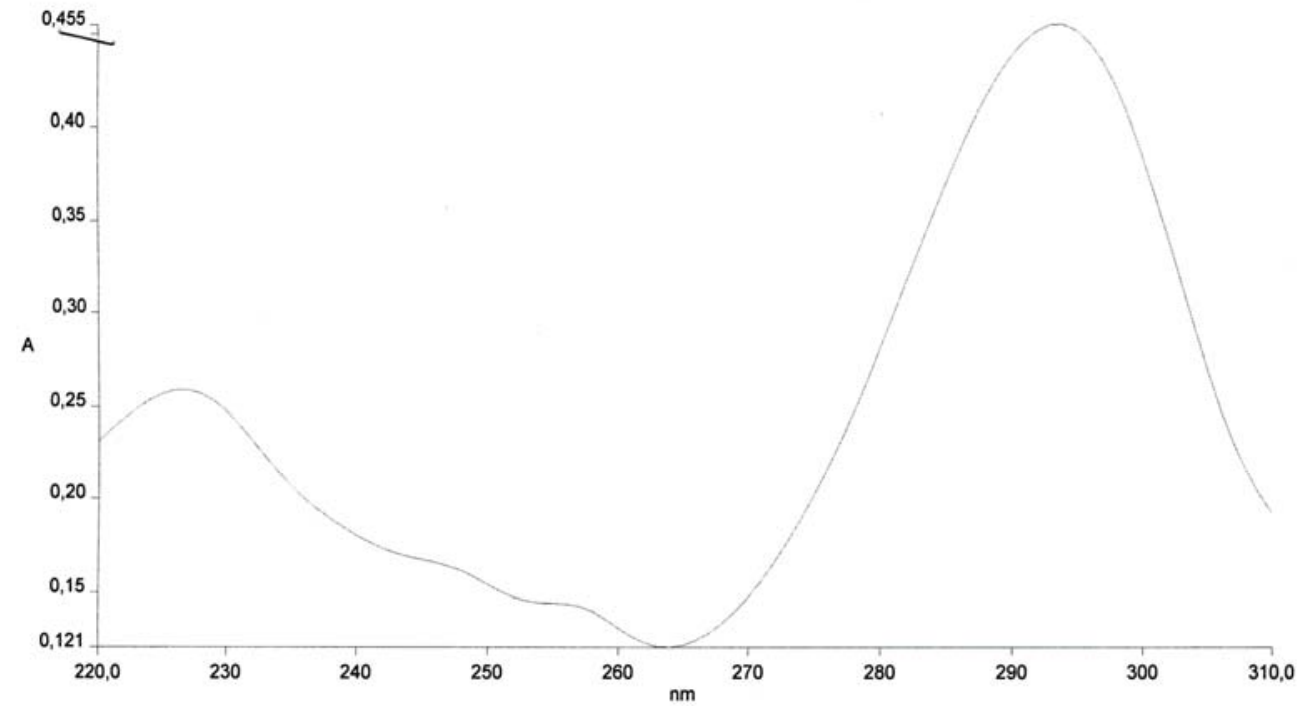

(c)

Fig. 4 a. UV absorption spectrum of levofloxacin, b. UV absorption spectrum of levofloxacin with silver nanoparticles $(\mathbf{0 . 0 0 1 1 9 \% )}$, c. UV absorption spectrum of levofloxacin with silver nanoparticles $(\mathbf{0 . 0 0 4 7 6 \% )}$.

\section{Conclusions}

Invariance of structures of metronidazole and levofloxacin after application on their surface silver nanoparticles by Electron-Ray Nanotechnology method makes it possible to use pharmacopoeial methods for quality control of metronidazole and levofloxacin and their nanocompositions with silver nanoparticles.

UV absorption spectra of solutions of metronidazole and metronidazole with silver nanoparticles and respectively solutions of levofloxacin and levofloxacin with silver nanoparticles have the same maxima and minima of absorption that shows the possibility of using ultraviolet spectrophotometry for identification and quantification both for metronidazole and levofloxacin, and their nanocompositions with silver nanoparticles.

For qualitative and quantitative determination of silver nanoparticles in metal-organic nanomaterials quality control methods should include additional methods, such as inductively coupled plasma atomic 

as the Basis for the Development of Quality Control Methods

emission spectroscopy method.

\section{References}

[1] Chopra, I. 2007. "The Increasing Use of Silver-based Products as Antimicrobial Agents: Useful Development or a Cause for Concern?.” Antimicrob Chem. 59: 587-90.

[2] Ruden, S., Hilpert, K., and Berditsch, M. 2009. "Synergistic Interaction between Silver Nanoparticles and Membrane-Permeabilizing Antimicrobial Peptides." Antib. Agents Chemoth 3538-40.

[3] Jain, J., Arora, S., and Rajwade, J. 2009. "Silver Nanoparticles in Therapeutics: Development of an Antimicrobial Gel Formulation for Topical Use." Mol. Pharm. 6: 1388-401.

[4] Sousa, A., Mehta, D., and Leavitt, R. 2006. "Bactericidal Activity of Combinations of Silver-Water Dispersion ${ }^{\mathrm{TM}}$ with 19 Antibiotics Against Seven Microbial Strains." Current Science 91: 926-9.

[5] Chekman, I. 2011. Nanopharmacology. Kiev: Zadruga. (in Ukrainian)

[6] Bilous, S. 2013. "Pharmaceutical Development of Antimicrobial Preparations with Silver Nanoparticles." Presented at XXII Scientific Congress of Polish Pharmaceutical Society, Bialystok, Poland.

[7] Bilous, S. B., and Kalynyuk, T. H. 2012. "Methodological Approaches to the Study of
Pharmaceutical Drug Development of Nanoscale Ingredients." Clin. Pharm. Pharmacoth Med. Standard 1-2: 95-101. (in Ukrainian)

[8] Paton, B., Moskalenko, V., and Chekman, I. 2009. "Nanoscience and Nanotechnology: Technical, Medical and Social Aspects." Bulletin NAS Ukraine 6: 18-26. (in Ukrainian)

[9] Movchan B. 2007. "Electron-ray Technology and New Materials in Medicine-First Steps.” Bulletin Pharmacol Pharmacy 12: 5-15. (in Ukrainian)

[10] Bond, A. D., and Jones, W. 2002. "Structure Prediction as a Tool for Solution of the Crystal Structures of Metallo-Organic Complexes Using Powder X-ray Diffraction Data." Asta Cryst B58: 233-43.

[11] David, W., and Shankland, K. 2008. "Structure Determination from Powder Diffraction Data." Asta Cryst A64: 52-64.

[12] Bruning, J., and Schmidt, M. U. 2015. "The Determination of Crystal Structures of Active Pharmaceutical Ingredients from X-ray Powder Diffraction Data: A Brief, Practical Introduction, with Fexofenadine Hydrochloride as Example." J. Pharm. Pharmacol. 67: 773-81.

[13] Bilous, S. B. 2015. "Algorithm of Nanosized Substances Quality Control Methods Development." Presented at 3rd International Conference on Pharmaceutical Sciences, Tbilisi, Georgia. 